\title{
Expression of cyclin D1 and cyclin $E$ in urothelial bladder carcinoma detected in tissue chips using a quantum dot immunofluorescence technique
}

\author{
GUANG SHAN $^{1}$ and TIAN TANG ${ }^{2}$
}

Departments of ${ }^{1}$ Urologic Surgery and ${ }^{2}$ Oncology, Renmin Hospital of Wuhan University, Wuhan, Hubei 430060, P.R. China

Received June 10, 2014; Accepted March 25, 2015

DOI: $10.3892 / 01.2015 .3436$

\begin{abstract}
The present study aimed to investigate the expression of cyclin D1 and cyclin E in tissue chips of bladder cancer using quantum dots (QDs), as well as examine its clinicopathological significance. The QD-based immunofluorescence tissue chemical technique was adopted to detect cyclin D1 and cyclin E expression in 75 tissue chips of human urothelial bladder carcinoma (including 70 cases of urothelial bladder carcinoma and 5 cases of cystitis), and its correlation with clinicopathological features was analyzed. The positive rates of cyclin D1 and cyclin E expression in urothelial bladder carcinoma were $68.6 \%$ (48/70) and 70.0\% (49/70), respectively; however, no expression was observed in cystitis. Based on the results of statistical analysis, the difference in the positive rates of cyclin D1 and cyclin E expression between urothelial bladder carcinoma and cystitis was significant $(\mathrm{P}<0.05)$. QD staining and statistical analysis revealed that the expression of cyclin D1 and cyclin E in urothelial bladder carcinoma was significantly higher compared with that in cystitis $(\mathrm{P}<0.05)$. However, no statistically significant difference $(\mathrm{P}>0.05)$ in cyclin $\mathrm{D} 1$ expression was observed in relation to pathological stage, clinical stage or invasion of urothelial bladder carcinoma; however, there was a significant difference $(\mathrm{P}<0.05)$ in cyclin $\mathrm{E}$ expression with respect to these factors. These results demonstrated that overexpression of cyclin D1 may be an early event in the occurrence of urothelial bladder carcinoma. Cyclin D1 may play a role in the initial stage where cell proliferation is a necessary step, without invasion or metastasis. In addition, overexpression of cyclin E was correlated with the stage and depth of invasion of urothelial bladder carcinoma. In conclusion, the abnormal expression of cyclin D1 and cyclin E may be involved in the occurrence and development of urothelial bladder carcinoma.
\end{abstract}

Correspondence to: Dr Guang Shan, Department of Urologic Surgery, Renmin Hospital of Wuhan University, 99 Ziyang Road, Wuhan, Hubei 430060, P.R. China

E-mail: gsttcn@163.com

Key words: urothelial bladder carcinoma, cyclin D1, cyclin E, tissue chip, quantum dot

\section{Introduction}

Bladder cancer is the most common malignant tumor of the urinary tract, with $95 \%$ of bladder cancer patients suffering from urothelial bladder carcinoma $(1,2)$. The rate of recurrence following surgical therapy is fairly high, and its incidence and mortality rates rank first among malignant tumors of the urinary system (3). Tumor invasion and metastasis is the main cause of mortality in patients, although the pathogenesis has not been fully elucidated. The occurrence and development of bladder cancer are known to be associated with multiple genes and multi-level cell signaling network disorder (4). The authors of the present study have previously conducted research into how genes and multi-level factors are associated with bladder cancer cell signaling network disorders (5-10); however, the research was not thorough on the subject of cell cycle protein with invasion and metastasis of tumors.

Dysregulation of the cell cycle is a key mechanism for the occurrence of tumors. Cyclin D1 and cyclin E have a key role in the regulation of the cell cycle and are a critical target of proliferative signals in $\mathrm{G}_{1}$ phase. A high expression of cyclin $\mathrm{E}$ shortens the $G_{1}$ phase and induces tumor development (11). By binding with cyclin-dependent kinase 4 (CDK4), cyclin D1 is activated, the compound CDK4-cyclin D1 is formed and the cell cycle is positively regulated. Patients with tumors in which cyclin D1 is overexpressed have a significantly lower survival rate compared with patients exhibiting a low expression of cyclin D1 (12). Therefore, cyclin D1 is an effective prognostic factor.

Cyclin E, as the key factor in regulating the transformation between phases $\mathrm{G}_{1}$ and $\mathrm{S}$, forms a compound with CDK2 and enters the $S$ phase (13). Research has revealed that the expression of cyclin $\mathrm{E}$ is in direct proportion to the proliferative activity of tumor cells, and is associated with poor prognosis of tumors $(14,15)$.

In the present study, a QD-based immunofluorescence tissue chemical technique was adopted in order to detect cyclin D1 and cyclin E expression, occurrence and development in urothelial bladder carcinoma, and evaluate the association between these factors using clinical pathology. This method serves to provide objective indicators for determining tumor invasion, metastasis and prognosis of urothelial bladder carcinoma. 


\section{Materials and methods}

Materials. In total, four urothelial bladder carcinoma chips were provided by Fanpu Biotech, Inc. (Guilin, China), which had a dot matrix of $15 \times 10$, a dot diameter of $1.1 \mathrm{~mm}$ and a thickness of $4 \mu \mathrm{m}$. The chips included 70 cases of urothelial bladder carcinoma and 5 cases of cystitis in a dual-chip matrix. The tissue samples (Fanpu Biotech, Inc.) were surgically resected during surgery and fixed for $24 \mathrm{~h}$ with $10 \%$ neutral-buffered formaldehyde. Among the patients with urothelial bladder carcinoma, there were 42 males and 28 females, aged between 26 and 80 years (mean age, 56.8 years). The cystitis samples were used as the control. This study was conducted in accordance with the Declaration of Helsinki and with the approval of the Ethics Committee of Wuhan University (Wuhan, China). Written informed consent was obtained from all the participants.

Patient classification. According to the 2004 World Health Organization classification of urothelial tumor histological types (16), 42 cases were confirmed as invasive urothelial carcinoma, 22 cases as high-grade non-invasive papillary urothelial carcinoma and 6 cases as low-grade non-invasive papillary urothelial carcinoma. According to the Union for International Cancer Control (UICC) clinical stages (17), 38 cases were classified as stage T1,22 cases as stage T2 and 10 cases as stage T3-4.

Reagents. Monoclonal rabbit anti-human cyclin D1 (cat. no. ZA-0101) and monoclonal mouse anti-human cyclin E (cat. no. ZM-0086) antibodies were obtained from Santa Cruz Biotechnology, Inc., (Dallas, TX, USA) at a dilution of 1:150. Secondary biotinylated goat anti-mouse and goat anti-rabbit immunoglobulin $G$ antibodies (cat. no. sfk12000) were purchased from Santa Cruz Biotechnology, Inc., at a dilution of $1: 300$

Experimental methods. The QD double-staining method was employed to detect cyclin D1 and cyclin E expression in the tissue chips of urothelial bladder carcinoma. The experiment was conducted in strict accordance with the instruction manual (18). The tissue chips of urothelial bladder carcinoma (thickness, $4 \mu \mathrm{m}$ ) were dewaxed and hydrated, then microwaved for antigen retrieval and washed in Tris-buffered saline (TBS) (17). The chips were blocked by incubation with blocking buffer solution (Wuhan Jiayuan Quantum Dot Technological Development Co., Ltd.) in a wet chamber for $30 \mathrm{~min}$ at $37^{\circ} \mathrm{C}$. Cyclin D1 and cyclin E antibodies were dripped into the chamber and incubated for $2 \mathrm{~h}$ at $37^{\circ} \mathrm{C}$, then washed three times in TBS-Tween for $5 \mathrm{~min}$. The chips were then incubated with blocking buffer solution in the wet chamber for $10 \mathrm{~min}$ at $37^{\circ} \mathrm{C}$. Next, biotinylated goat anti-mouse or goat anti-rabbit immunoglobulin $\mathrm{G}$ antibody (cat. no. sfk12000; Santa Cruz Biotechnology, Inc.) was added to the wet chamber and incubated for $20 \mathrm{~min}$ at $37^{\circ} \mathrm{C}$. QDs-SA diluted with the blocking buffer solution was dripped into the wet chamber and incubated for $30 \mathrm{~min}$ at $37^{\circ} \mathrm{C}$, followed by washing with TBS-Tween three times for $5 \mathrm{~min}$ each time and then adding 90\% glycerin buffer (19). Then, a fluorescence microscope (DM2700 m; Leica Microsystems GmbH, Wetzlar, Germany) was used to observe the tissue chips. Upon observation under the microscope, the cells presenting with green fluorescence were considered to exhibit a positive expression of cyclin E, while cells presenting with red fluorescence were considered to exhibit a positive expression of cyclin D1. A positive area of $\geq 25 \%$ indicated that the cells exhibited a positive expression. In the control group, the primary antibody was substituted with TBS to form the control group, and the available positive chip was used as the positive control.

Quantitative analysis of the cyclin D1 and cyclin E expression levels. The NuanceFX ${ }^{\mathrm{TM}}$ multi-spectral imaging system (PerkinElmer, Inc., Waltham, MA, USA) was employed to perform quantitative analysis of the expression of cyclin D1 and cyclin E. Five complete and non-overlapping views were randomly selected under a high-power lens (magnification, $\mathrm{x} 400$ ). The mean optical density, positive area and total area of all cells with a positive reaction under each view were measured, and the ratio of the positive area was calculated. The mean value of the optical density and positive area per case was used as the measured value of the case (ratio of positive area $=$ total area of positive reaction per unit / total area of cells per unit x 100\%).

Statistical analysis. The results of QD staining are expressed as the mean \pm standard deviation. One-way analysis of variance and the Student-Neuman-Keuls q-test were conducted using the SPSS 13.0 software (SPSS Inc., Chicago, IL, USA) to confirm the mean optical density and ratio of the positive area in each group $(\alpha=0.05)$. Prior to detection, a normality test and homogeneity test of variances were performed. $\mathrm{P}<0.05$ was considered to indicate a statistically significant difference..

\section{Results}

Expression of cyclin D1. With regard to positive cyclin D1 expression, strong red fluorescence was observed mainly in the cytoplasm of tumor cells, indicating that cyclin D1 was highly expressed (Fig. 1). However, cyclin D1 expression was not observed in cystitis tissues (Fig. 2). In total, 48 cases (68.6\%) of urothelial bladder carcinoma demonstrated positive cyclin D1 expression. The average optical density and the positive area rate were found to be significantly different between the urothelial bladder cancer and cystitis tissues $(\mathrm{P}<0.05)$. The results of the image analysis are listed in Table I. In addition, no statistically significant difference in cyclin $\mathrm{D} 1$ expression $(\mathrm{P}>0.05)$ in relation to patient age, gender, depth of invasion or clinical UICC stage, as shown in Table II.

Expression of cyclin E. With regard to positive cyclin D1 expression, strong green fluorescence was observed mainly in the cell nuclei of tumor cells (Fig. 1). By contrast, cyclin E expression was not observed in cystitis (Fig. 2). In total, 49 cases $(70 \%)$ of urothelial bladder carcinoma demonstrated positive cyclin E expression. The average optical density and the positive area rate were found to be significantly different between the urothelial bladder cancer and cystitis tissues $(\mathrm{P}<0.05)$. The results of the image analysis are listed in 
Table I. Quantum dot immunofluorescence staining used to detect average optical density and rate of positive area of cyclin D1.

\begin{tabular}{lrcrr}
\hline Group & $\mathrm{n}$ & Number of slices & Average optical density & Rate of positive area \\
\hline Urothelial bladder cancer & 70 & 350 & $0.5240 \pm 0.0341^{\mathrm{a}}$ & $0.4694 \pm 0.0310^{\mathrm{a}}$ \\
Cystitis tissue & 5 & 25 & $0.1397 \pm 0.0198$ & $0.1128 \pm 0.0167$ \\
\hline
\end{tabular}

${ }^{a} \mathrm{P}<0.05$ vs. cystitis tissue. Data are expressed as the mean \pm standard deviation.

Table II. Correlation of cyclin D1 and cyclin E expression with the pathological type and clinical stage of urothelial bladder carcinoma.

\begin{tabular}{|c|c|c|c|c|c|c|c|}
\hline \multirow[b]{2}{*}{ Pathological features } & \multirow[b]{2}{*}{$\mathrm{n}$} & \multicolumn{2}{|c|}{ Cyclin D1 } & \multirow[b]{2}{*}{ P-value } & \multicolumn{2}{|c|}{ Cyclin E } & \multirow[b]{2}{*}{ P-value } \\
\hline & & - & + & & - & + & \\
\hline Gender & & & & $>0.05$ & & & $>0.05$ \\
\hline Male & 42 & 14 & 28 & & 12 & 30 & \\
\hline Female & 28 & 8 & 20 & & 9 & 19 & \\
\hline Age (years) & & & & $>0.05$ & & & $>0.05$ \\
\hline$\leq 60$ & 37 & 10 & 27 & & 11 & 26 & \\
\hline$>60$ & 33 & 12 & 21 & & 10 & 23 & \\
\hline Depth of tumor invasion & & & & $>0.05$ & & & $<0.05$ \\
\hline Invasive & 42 & 18 & 24 & & 10 & 32 & \\
\hline Non-invasive high-grade & 22 & 10 & 12 & & 8 & 14 & \\
\hline Non-invasive low-grade & 6 & 2 & 4 & & 2 & 4 & \\
\hline UICC stage & & & & $>0.05$ & & & $<0.05$ \\
\hline $\mathrm{T} 1$ & 38 & 16 & 22 & & 10 & 28 & \\
\hline $\mathrm{T} 2$ & 22 & 8 & 14 & & 6 & 16 & \\
\hline T3-4 & 10 & 4 & 6 & & 2 & 8 & \\
\hline
\end{tabular}

UICC, Union for International Cancer Control.

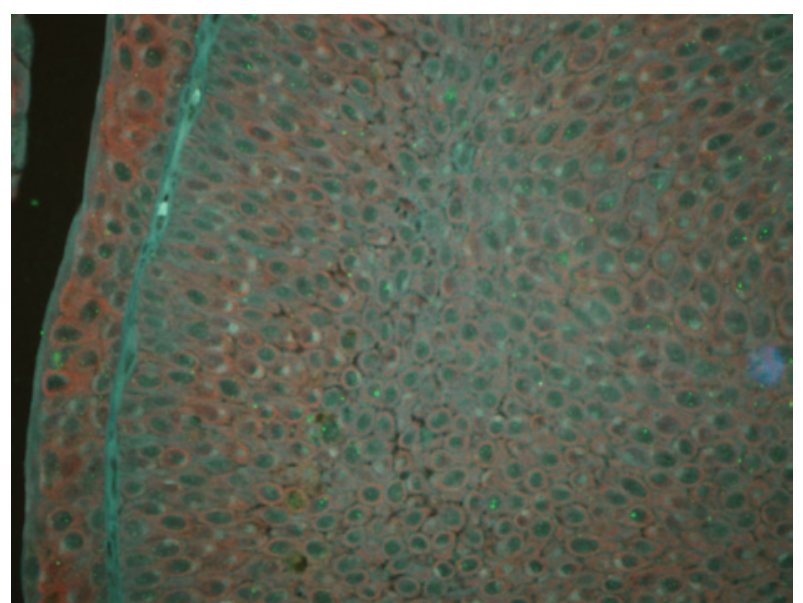

Figure 1. Expression of cyclin D1 and cyclin E in urothelial bladder cancer, detected using sensitive quantum dot double-staining (magnification, x200). Cyclin D1 (red fluorescence) and cyclin E (green fluorescence) were highly expressed in the urothelial bladder cancer tissues.

Table III. In addition, no statistically significant differences in cyclin E expression ( $\mathrm{P}>0.05)$ were observed in relation to patient age and gender. However, a significant difference in

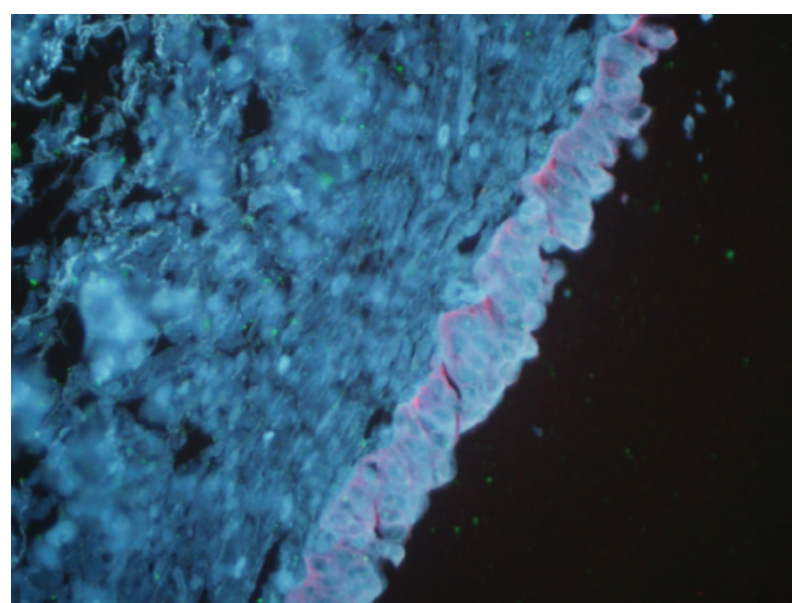

Figure 2. Expression of cyclin D1 and cyclin E in cystitis tissues, detected using sensitive quantum dot double-staining (magnification, x200). Cyclin D1 and cyclin $\mathrm{E}$ were not expressed in cystitis tissues.

the expression of cyclin $\mathrm{E}(\mathrm{P}<0.05)$ was detected in relation to the depth of invasion and clinical UICC stage, as demonstrated in Table II. 
Table III. Quantum dot immunofluorescence staining used to detect average optical density and rate of positive area of cyclin E.

\begin{tabular}{lrcrr}
\hline Group & $\mathrm{n}$ & Number of slices & Average optical density & Rate of positive area \\
\hline Urothelial bladder cancer & 70 & 350 & $0.4988 \pm 0.0320^{\mathrm{a}}$ & $0.5102 \pm 0.0341^{\mathrm{a}}$ \\
Cystitis tissue & 5 & 25 & $0.1547 \pm 0.0189$ & $0.1395 \pm 0.0126$ \\
\hline
\end{tabular}

${ }^{\mathrm{a}} \mathrm{P}<0.05$ vs. cystitis tissue. Data are expressed as the mean \pm standard deviation.

Correlation between cyclin DI and cyclin E expression. Considering the aforementioned observations, the cyclin D1 and cyclin E expression was found to be significantly and positively correlated in the 70 cases of urothelial bladder carcinoma.

\section{Discussion}

Bladder cancer is the most common malignant tumor of the urinary tract, with $\sim 95 \%$ of bladder cancer patients suffering from urothelial bladder carcinoma $(20,21)$. Approximately $80 \%$ of bladder cancer cases are superficial in the early stage, and $70 \%$ of such patients suffer relapse following surgery, with $30 \%$ of relapses developed towards the later stages of the disease (1). In addition, 15-30\% of cases are invasive at the early stage, even if therapy is received for early metastasis (22). The prognosis of patients with urothelial bladder carcinoma is determined by the depth of invasion, metastasis and recurrence. Therefore, the study of factors, including tumor invasion and metastasis of urothelial bladder carcinoma, is of great clinical significance. Invasion and metastasis of tumors are the main causes of mortality, although the pathogenesis has not been fully elucidated $(23,24)$.

The occurrence and development of bladder cancer are known to be connected with multiple genes and multi-level cell signaling network disorder (4). Previous research into how genes and multi-level factors are associated with bladder cancer cell signaling network disorders has been conducted by the current authors (5-10); however, the research was not thorough on the subject of cell cycle protein with invasion and metastasis of tumors. Further investigating the underlying mechanism of the occurrence and development of bladder cancer, as well as searching for effective indicators to predict its biological behavior, is crucial in order to assist its early diagnosis and provide an experimental basis for guiding treatment and prognosis. Studying the association between the occurrence of tumors and the cell cycle progression is currently a hotspot in tumor biology research (25). Cyclin D1 and cyclin E are significant regulatory factors in the $G_{1}$ and $S$ phases of the cell cycle; however, their correlation with bladder cancer remains unclear.

A tissue chip (or tissue microarray) refers to a tissue section consisting of tens of thousands of tissue blocks that are neatly placed on a glass slide. Tissue chips have a small volume, but contain a large quantity of information, demonstrating substantial results in one-time experiments. Data with regard to cyclin D1 and cyclin E expression in urothelial bladder carcinoma may be obtained in a short period of time. In contrast to traditional pathological techniques and methods, the results obtained using the tissue microarray technique are consistent, reliable and fairly comparable, which can reduce time and cost, and provide a large quantity of data. This method has several promising applications. Tissue chips may be used in fields including basic, clinical and application research, as well as medicine development, and has promising prospects for development (10).

QDs are novel fluorescence semiconductor nanocrystals, which present a broad and continuous spectrum, high absorptivity and intensity, a narrow and symmetrical emission peak, and fast light bleaching, compared with the traditional organic fluorescence labeling reagents (26-28). QDs are able to withstand repetitive activation, have activated pathology and may be applied in tumor diagnosis and imaging studies (29).

In the present study, the QD immunofluorescence and tissue chip techniques were adopted to detect cyclin D1 and cyclin E expression, occurrence and development in human urothelial bladder carcinoma. In addition, the association of their expression with clinicopathological factors was investigated in order to improve the understanding on the molecular mechanism of occurrence and development of urothelial bladder carcinoma, as well as its biological behavior.

The regulation of cell cycle progression mainly relies on the formation, activation and interaction of cyclin, CDK and CDK inhibitor. As key positive regulatory factors, cyclin D1 and cyclin $E$ play critical roles in $G_{1}$ phase regulation and $\mathrm{G}_{1} / \mathrm{S}$ phase transformation. Cyclin $\mathrm{D} 1$ has been demonstrated to be a cancer gene that is directly associated with tumors. Cyclin D1 (11), as the most significant positive regulatory factor of the cell cycle, plays a key role in the occurrence and development of tumors (30). Under a physiological state (31), as the cell enters the S phase, cyclin D1 breaks down. In cases where the cyclin D1 gene is activated, cyclin D1 is highly and continuously expressed. $G_{1}$ phase is then shortened and cyclin D1 enters the $\mathrm{S}$ phase early; thus, cell proliferation is uncontrollable and the tumor is formed.

Cyclin $E$ is a positive regulatory factor in the $G_{1} / S$ phase transformation and regulation. It is synthesized after cyclin D, and kinase activity reaches its peak in the late period of $\mathrm{G}_{1}$ phase. However, the chromosomal localization of cyclin $\mathrm{E}$ gene remains unclear. In the body, cyclin $\mathrm{E}$ combines with CDKZ to form the compound cyclin E-CDKZ, which phosphorylates and deactivates the retinoblastoma protein (Rb) (32). Subsequently, the transcription factor inhibited by the phosphorylated $\mathrm{Rb}$ is released, while $\mathrm{G}_{1} / \mathrm{S}$ transformation and DNA synthesis are promoted, and cell proliferation is realized. After entering the $\mathrm{S}$ phase, cyclin E breaks down quickly. Cyclin D1 and cyclin E, as key rate-limiting factors in the $G_{1} / S$ transformation of the cell cycle, play significant roles in cell proliferation (33). In addition, cyclin $\mathrm{E}$ is considered as 
a backup cyclin in regulating $\mathrm{G}_{1} / \mathrm{S}$ phase transformation. As a cancer gene, cyclin E overexpression exerts harmful effects on the cell cycle, which may cause uncontrollable cell proliferation and promotion of tumor occurrence and development. As previously reported in the literature, cyclin E gene amplification and protein overexpression may be detected in human tumors, including breast, ovarian, gastric, esophageal, pancreatic and lung cancer (34). In addition, previous studies have identified that cyclin E overexpression is associated with the stage, grading, metastasis and life cycle of certain tumors, including breast cancer, gastric cancer, renal pelvic carcinoma and ureteral cancer (35-37). Therefore, cyclin E expression is considered to be a significant prognostic marker for these cancers.

In a number of tumor tissues, cyclin E generates gene amplification and overexpression, and has already been confirmed as a cancer gene (38). In the majority of tumor studies, the immunolabeling technique is used to determine cyclin E expression in the cell nucleus (39-42). Cyclin E protein is synthesized and degraded in the cytoplasm, and is generally quickly transferred to the cell nucleus (43). The accumulation of cyclin $\mathrm{E}$ in the cytoplasm may demonstrate increased synthesis, decreased degradation and nuclear transport disorder (32). Cyclin E is mainly engaged in cell division within the cell nucleus; therefore, tumor cells with negative nuclear and cytoplasm staining are considered as having negative expression (40).

In the present study, QD staining and statistical analysis were performed. According to the results, cyclin D1 expression in urothelial bladder carcinoma tissues was greater compared with that in cystitis tissues, and the difference was statistically significant $(\mathrm{P}<0.05)$. This indicates that cyclin $\mathrm{D} 1$ protein overexpression shortens the cell cycle of $\mathrm{G}_{1}$ phase, diminishes its volume and weakens its dependence on mitogen. Simultaneously, the overexpression results in uncontrolled cell proliferation and leads to urothelial bladder carcinoma development. Based on the current experimental results, cyclin D1 expression has no significant association with the clinicopathological stages of urothelial bladder carcinoma, indicating that cyclin D1 overexpression may be an early event in the occurrence of urothelial bladder carcinoma, and may be important in the initial stages where cell proliferation is a necessary step, involving no tumor invasion or metastasis.

Using QD staining, cyclin E expression was also detected in urothelial bladder carcinoma tissues, and statistical analysis was conducted. Cyclin E expression was observed to be higher in urothelial bladder carcinoma tissues compared with that in cystitis tissues, and the difference was statistically significant $(\mathrm{P}<0.05)$. In addition, cyclin E expression exhibited a significant association with the clinicopathological stages of urothelial bladder carcinoma $(\mathrm{P}<0.05)$. Therefore, cyclin $\mathrm{E}$ may participate in the occurrence, development and metastasis of urothelial bladder carcinoma and may be a significant reason for the active proliferation of tumor cells. The present study, reporting the overexpression of cyclin D and cyclin E in urothelial bladder carcinoma, may assist the investigation into the pathogenesis of this disease and the classification of the disease into clinical stages and degrees of malignancy, thus serving as a novel therapeutic approach.

In conclusion, cyclin D1 and cyclin E are key regulatory factors of $G_{1} / S$ phase transformation. Their overexpression shortens the duration of $\mathrm{G}_{1} / \mathrm{S}$ phase transformation and promotes the progression of the cell cycle and increased cell proliferation, which results in tumorigenesis. The synergetic effect of their overexpression may affect the occurrence and development of urothelial bladder carcinoma. Further investigation on the correlation between cyclin and urothelial bladder carcinoma is likely to provide an insight into the mechanism of tumorigenesis. Previous studies have confirmed that cyclin plays a significant role in the occurrence of tumors and is able to provide prognostic information on a number of common tumors $(35,44)$. However, the underlying mechanism through which cyclin affects tumor development remains unclear and requires further elucidation.

\section{References}

1. Jemal A, Siegel R, Xu J and Ward E: Cancer statistics, 2010. CA Cancer J Clin 60: 277-300, 2010.

2. Friedrich MG, Weisenberger DJ, Cheng JC, et al: Detection of methylated apoptosis-associated genes in urine sediments of bladder cancer patients. Clin Cancer Res 10: 7457-7465, 2004.

3. Yakasai A, Allam M and Thompson AJ: Incidence of bladder cancer in a one-stop clinic. Ann Afr Med 10: 112-114, 2011.

4. Vlahou A: Back to the future in bladder cancer research. Expert Rev Proteomics 8: 295-297, 2011

5. Shan G and Xia Y: Expression and clinical significance of RECK and MT1-MMP in bladder urothelium carcinoma tissues. Chin J Cancer Prev Treat 19: 1722-1725, 2012.

6. Shan G, Shan S, Zhang X and Liu XH: Expression and clinical sigificance of cyclin G1 and cyclin G2 in transitional cell carcinoma of bladder. Chinese J Histochem Cytochem 18: 268-273, 2009.

7. Shan G and Tang T: Expression and clinical sigificance of ADO in transitional cell carcinoma of bladder. Chinese J Histochem Cytochem 20: 267-271, 2011.

8. Shan G and Tang T: Expression and clinical sigificance of tumor suppressor genes DPC4 and TGF- $\beta 1$ in transitional cell carcinoma of bladder. Chinese J Histochem Cytochem 20: 491-495, 2011.

9. Shan G and Tang T: Expression and clinical sigificance of CD82/KAI1 in transitional cell carcinoma of bladder. Chinese J Histochem Cytochem 22: 185-188, 2013.

10. Shan $G$ and Tang T: Expression and clinical significance of PSCA and mesothelin in transitional cell carcinoma of bladder. Chinese J Histochem Cytochem 6: 684-692, 2010.

11. Otieno S, Grace CR and Kriwacki RW: The role of the LH subdomain in the function of the Cip/Kip cyclin-dependent kinase regulators. Biophys J 100: 2486-2494, 2011.

12. Jirawatnotai S, Hu Y, Michowski W, et al: A function for cyclin D1 in DNA repair uncovered by protein interactome analyses in human cancers. Nature 474: 230-234, 2011.

13. Makiyama K, Masuda M, Takano Y, et al: Cyclin E overexpression in transitional cell carcinoma of the bladder. Cancer Lett 151: 193-198, 2000.

14. Farley J, Smith LM, Darcy KM, et al; Gynecologic Oncology Group: Cyclin E expression is a signifieant predictor of survival in advanced, suboptimally debulked ovarian epithelial cancers: a Gynecologic Oncology Group study. Cancer Res 63: 1235-1241, 2003.

15. Scuderi R, Palucka KA, Pokorvskaja K, et al: Cyclin E overexpression in relapsed adult acute lymphoblastic leukemias of B-cell lineage. Blood 87: 3360-3367, 1996.

16. Eble JN, Sauter G, Epstein JI and Sesterhenn IA (eds): World Health Organization Classification of Tumors. Pathology and Genetics of Tumors of the Urinary System and Male Genital Organs. IARC Press, Lyon, 2004.

17. Sobin LH, Gospodarowicz M and Wittekind C (eds). Urological tumours. In: TNM Classification of Malignant Tumors. UICC International Union Against Cancer. 7th edition. Wiley-Blackwell, pp262-265, 2009.

18. Chen H, Xue J, Zhang Y, Zhu X, Gao J and Yu B: Comparison of quantum dots immunofluorescence histochemistry and conventional immunohistochemistry for the detection of caveolin-1 and PCNA in the lung cancer tissue microarray. J Mol Hist 40: 261-268, 2009. 
19. Tang T and Zhang DL: Study on extracellular matrix metalloproteinase inducer and human epidermal growth factor receptor-2 protein expression in papillary thyroid carcinoma using a quantum dot-based immunofluorescence technique. Exp Ther Med 9: 1331-1335, 2015.

20. Parkin DM, Bray F, Ferlay J and Pisani P: Global cancer statistics, 2002. CA Cancer J Clin 55: 74-108, 2005.

21. Jemal A, Siegel R, Ward E, et al: Cancer statistics, 2008. CA Cancer J Clin 58: 71-96, 2008.

22. Dhawan D, Ramos-Vara JA, Naughton JF, et al: Targeting folate receptors to treat invasive urinary bladder cancer. Cancer Res 73: 875-884, 2013.

23. Sylvester RJ, van der Meijden AP, Oosterlinck W, et al: Predicting recurrence and progression in individual patients with stage Ta T1 bladder cancer using EORTC risk tables: A combined analysis of 2596 patients from seven EORTC trials. Eur Urol 49: 466-475; discussion, 475-477, 2006.

24. Amit D, Tamir S, Birman T, et al: Development of targeted therapy for bladder cancer mediated by a double promoter plasmid expressing diphtheria toxin under the control of IGF2-P3 and IGF2-P4 regulatory sequences. Int J Clin Exp Med 4: 91-102, 2011.

25. Das SN, Khare P, Singh MK and Sharma SC: Correlation of cyclin D1 expression with aggressive DNA pattern in patients with tobacco-related intraoral squamous cell carcinoma. Indian J Med Res 133: 381-386, 2011.

26. Remacle F and Levine RD: Quantum dots as chemical building blocks: elementary theoretical considerations. Chemphyschem 2: 20-36, 2001.

27. Yu WW, Chang E, Drezek R and Colvin VL: Water-soluble quantum dots for biomedical applications. Biochem Biophys Res Commun 348: 781-786, 2006.

28. Koole R, Mulder WJ, van Schooneveld MM, Strijkers GJ, Meijerink A and Nicolay K: Magnetic quantum dots for multimodal imaging. Wiley Interdiscip Rev Nanomed Nanobiotechnol 1: 475-491, 2009.

29. Li J, Huang X, Xie X, Wang J and Duan M: Human telomerase reverse transcriptase regulates cyclin $\mathrm{D} 1$ and $\mathrm{G}_{1} / \mathrm{S}$ phase transition in laryngeal squamous carcinoma. Acta Otolaryngol 131: 546-551, 2011.

30. Hwang CF, Cho CL, Huang CC, et al: Loss of cyclin D1 and p16 expression correlates with local recurrence in nasopharyngeal carcinoma following radiotherapy. Ann Oncol 13: 1246-1251, 2002.
31. Umekita Y, Ohi Y, Sagara Y and Yoshida H: Over expression of cyclin D1 predicts for poor prognosis in estrogen receptor-negative breast cancer patients. Int J Cancer 98: 415-418, 2002.

32. Stamatakos M, Palla V, Karaiskos I, et al: Cell cyclins: triggering elements of cancer or not? World J Surg Oncol 8: 111, 2010.

33. Freemantle SJ and Dmitrovsky E: Cyclin E transgenic mice: discovery tools for lung cancer biology, therapy and prevention. Cancer Prev Res (Phila) 3: 1513-1518, 2010.

34. Musat M, Morris DG, Korbonits M and Grossman AB: Cyclins and their related proteins in pituitary tumourigenesis. Mol Cell Endocrinol 326: 25-29, 2010

35. Jung YJ, Lee KH, Choi DW, et al: Reciprocal expressions of cyclin E and cyclin D1 in hepatocellular carcinoma. Cancer Lett 168: 57-63, 2001.

36. Pajalunga D and Crescenzi M: Regulation of cyclin E protein levels through E2F-mediated inhibition of degradation. Cell Cycle 3: 1572-1578, 2004.

37. Richter J, Wagner U, Kononen J, et al: High-throughput tissue microarray analysis of cyclin E gene amplification and overexpression in urinary bladder cancer. Am J Pathol 157: 787-794, 2000.

38. Li JQ, Miki H, Ohmori M, et al: Expression of cyclin E and cyclin-dependent kinase 2 correlates with metastasis and prognosis in colorectal carcinoma. Hum Pathol 32: 945-953, 2001.

39. Donnellan R, Kleinschmidt I and Chetty R: Cyclin E immunoexpression in breast ductal cancinoma: pathologic correlations and prognostic implications. Hum Pathol 32: 89-94, 2001.

40. Zhu HX: Cyclin E and tumor. Chinese Medical Journal of Zhong Guo Yejin Gongye Yixue Zazhi 20: 86-88, 2003 (In Chinese).

41. Bales ES, Dietrich C, Bandyopadhyay D, et al: High levels of expression of $\mathrm{p} 27 \mathrm{KIPl}$ and cyclin $\mathrm{E}$ in invasive primary malignant melanomas. J Invest Dermatol 113: 1039-1046, 1999.

42. Harwell RM, Porter DC, Danes C and Keyomarsi K: Processing of cyclin E differs between normal and tumor breast cells. Cancer Res 60: 481-489, 2000.

43. Siu KT, Rosner MR and Minella AC: An integrated view of cyclin E function and regulation. Cell Cycle 11: 57-64, 2012.

44. Bassiouny AE, Nosseir MM, Zoheiry MK, et al: Differential expression of cell cycle regulators in $\mathrm{HCV}$-infection and related hepatocellular carcinoma. World J Hepatol 2: 32-41, 2010. 